\title{
SIX FAILURES OF THE DOHERTY MODELLING REPORT
}

\section{A PREPRINT}

\author{
Jeremy P. Howard \\ University of San Francisco \\ Kalinda E Griffiths \\ University of New South Wales \\ Rachel L. Thomas \\ Queensland University of Technology
}

September 7, 2021

\begin{abstract}
The Doherty Model is being used in Australia to justify partial reopening with $70 \%$ of adults vaccinated [Doherty Institute, 2021]. However, we have identified six critical failures of the model: failure to model uncertainties; failure to use appropriate premises; failure to model subgroup vaccine takeup; failure to correctly model child transmission; failure to include relevant outcomes; and failure to consider longer time-frames. These failures result in missing over 200,000 cases of long covid in children, underestimating death counts by a factor of up to ten, underestimating the severity of the delta variant by a factor of two, and greatly underestimating the potential downside risk.
\end{abstract}

Keywords SARS-CoV-2, Modeling, Epidemiology

\section{Background}

The Australian federal government, and some state and territory governments, are calling for loosening covid restrictions at $70 \%$ of adults vaccinated, and is using a model known as the Doherty Model to justify this [Doherty Institute, 2021].

The gold standard for models is open source code and open data [Huston et al,, 2019]. That means that everything in the model is open to peer review from the full scientific community. However, much of the modeling and data for the Doherty Model is secret.

Modeling has been used around the world to help create, and justify, health policy in response to covid. However, much of it has been wildly wrong, and it has often taken global collaborations of scientists many months to find and correct critical problems [Cepelewicz, 2021]. There is no reason to believe that the Doherty Model would not have similar issues. Since Australian scientists can't study it directly, we just do not know.

Here we study the reports which describe the model, and consider how to interpret the model on the assumption that it correctly implements that description.

\section{Failure to model uncertainties}

The Doherty Model has a very challenging job. It has to work with incomplete data, such as the very limited information we have about the behavior of the delta variant, and it has to make guesses about things that are extremely hard to predict, such as the behavior of humans.

The recommended practice in this kind of situation is to not make a single assumption about the premises in a model, but to instead model uncertainty, by including a range of possible values for each uncertain premise [Greenland, 2004, MacLehose and Hamra, 2014]. For instance, we do not really know how severe the delta variant is. So instead of 
making a single guess about severity, modelers should consider the range of possible values based on the information available. Then the model is re-run many times, each time using a different set of premises from the chosen ranges. The output of such a model is a range of possible outcomes, along with an estimate of the probability of each.

The Doherty Model does not do this. Instead, point estimates, that is, a single guess for each premise, are used. And a single output is produced by the model. A small number of scenarios are shown, for a range of assumptions about vaccine rates, and for two levels of contact tracing effectiveness. But the vast majority of assumptions are only included as point estimates. This is a critical deficiency. By failing to account for uncertainty in inputs, the model also fails to account for uncertainty in outputs. The Doherty Model claims that Australia will have around a thousand covid deaths over the next six months. But what is the probability that there is a hundred times more than that? What is the probability that the hospitalizations are far more rapid than in their single modeled outcome, such that Australian ICUs are overloaded? We do not know, because that work has not been done.

\section{Failure to use appropriate premises}

The Doherty Model has to work with incomplete data. In such a situation, modelers should aim to estimate model parameters which are in the middle of reasonable bounds, rather than choosing an extreme value. A common error is to assume that in the absence of clear data to the contrary, that zero should be chosen for parameters where data is scarce. However, this is clearly at odds with the above guideline: zero is an extreme value, and should only be used where there is good reason to believe that is the most reasonable estimate.

The Doherty Model makes this error in a critical place: the report says we will assume that the severity of Delta strains approximates Alpha strains. No evidence is provided to support this extreme assumption. We now know that it is incorrect: latest estimates are that People who are infected with the highly contagious Delta variant are twice as likely to be hospitalized as those who are infected with the Alpha variant.

A similar error is made in the premises used for Test, Trace, Isolate, and Quarantine (TTIQ) capability in the model. The model looks at two scenarios, optimal, where contact tracing and isolation is so effective that nearly all transmission is stopped, and partial, which is described as being just for situations where there are thousands of cases. However the partial scenario is based on the experience in Victoria when there were 700 cases, not thousands.

Key population characteristics of missing subgroups are not included in the modelling, although rurality/remoteness is acknowledged as a future consideration in the report [Doherty Institute, 2021]. As case numbers grow in regional and remote Australia, the inclusion of parameters that recognise age variations in sub-populations can affect the recommendations arising. This is of particular importance for Indigenous people, whose age distribution is much younger than the general population with the median age being 23 years compared to 37.8 years for non-Indigenous people [Australian Bureau of Statistics, 2018].

\section{Failure to model subgroup vaccine takeup}

The Doherty Model assumes that vaccines are equally distributed throughout the country, within each age group. However, there are groups where thats not true. For instance, some Indigenous communities are largely unvaccinated and there is variability in times between the first and second vaccinations due to access to services. Additionally, Indigenous people experience high rates of overcrowding where people in non-urban areas are most affected, resulting in higher numbers of close contacts.

In an unvaccinated subpopulation which mixes regularly, in the absence of restrictions, then $\mathrm{R}$ will return towards 5.0 (the reproduction number of delta without vaccines or restrictions) [Fine et al, 2011]. At that reproduction rate, an outbreak of just 32 cases develops to 500,000 cases in under two months.

There are always pockets of lower vaccination rates in any population. By failing to model this, the Doherty model understates the risk to these groups.

\section{Failure to correctly model child transmission or severity}

The Doherty model fails to model school mixing, but instead makes a simplifying assumption that children have some random chance of meeting random other children each day. In practice however, they have a $100 \%$ chance of mixing with exactly the same children every day, at school. This makes a big difference to the path of the pandemic in children. Zhang et al. [2021] showed that nearly all children in a school population would be infected with the delta variant within a single semester. 


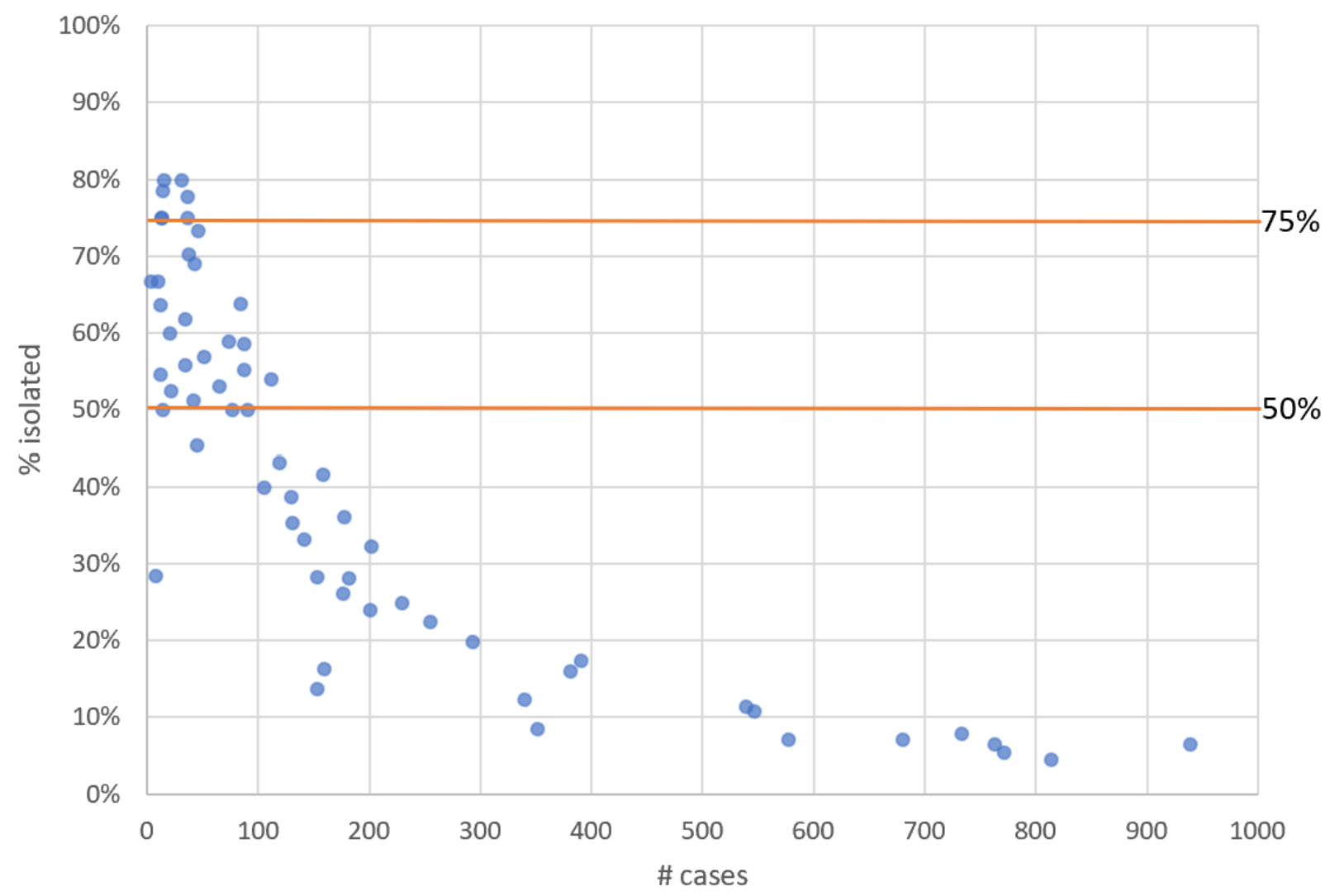

Figure 1: NSW TTIQ efficacy vs Number of Daily Cases

For modeling the impact of the pandemic in children, Doherty Institute [2(221] assumed that transmission and severity dynamics matched those used in Davies et an] [2020], which in turn relied on data from the Wuhan outbreak in January 2020 Surveillances [2020]. This data and analysis is far out of date. Testing methods used in January 2020 missed many cases, and the virus has been through multiple variants since then.Additionally, the modelling does not consider variability of severity in different subgroups. For example, Indigenous children have higher rates of obesity and chronic respiratory infections [Australian Institute of Health and Weltare, 2015].

\section{Failure to include relevant outcomes}

The Doherty report does not show the predicted number of cases, or the predicted incidence of long covid. Long covid is associated with many problems including long-term cognitive dysfunction, acceleration of Alzheimers symptoms [Alzheimer's Association, 2021], fatigue, shortness of breath, muscle ache, and difficulty concentrating, with symptoms adversely affecting the day-to-day activities of $66 \%$ of cases [Office for National Statistics, 2021].

By failing to include these outcomes, readers are unable to assess the full impact of the pandemic.

Although the number of cases is not reported directly, it can be calculated from the information provided. The number of symptomatic cases is shown, as is the percentage of cases that are symptomatic. From these, we can calculate that up to 1.4 million children would be infected based on the models assumptions.

Wise [2021] estimate that one in seven children will develop long covid. With 1.4 million infected children, that would result in 200,000 cases of long covid in Australian children, assuming that the Doherty model's count of infection of children is accurate. If the simulation of Zhang et al. [2021] is correct then there would be 600,000 long covid cases. If in addition the Delta variant is twice as severe for long covid as alpha, then there would be 1,200,000 long covid cases.

Failing to consider long covid, including in children, is a significant oversight in the report. 


\section{Failure to consider longer time frames}

The Doherty Model fails to account for time after 180 days, when most cases occur. This is the Doherty chart of cases if Australia hits $80 \%$ vaccine for all adults it stops after 180 days, even although the number of cases is still increasing:

[Hyde et al, 2021] estimated the full impact of covid without such a time limitation. They found that there would be around 25,000 deaths in Australia in the absence of restrictions. They recommend a 90\% of all Australians (including kids) vaccination target. This is also the recommendation from the Grattan Institute, who found we need to vaccinate at least $80 \%$ of the total population (including kids) this year, and continue the vaccination roll-out to $90 \%$ throughout 2022 Duckett et al, [2021]].

We can estimate the longer term impact of covid by looking at other countries. In the UK, $75 \%$ of $16+$ residents are vaccinated. There are currently 700 covid deaths and 250,000 cases per week in the UK. If our death rate is proportionate, that would mean 266 Australians dying per week even after we get to $75 \%$ vaccinated (along with thousands of long covid cases, with their huge economic and societal cost). By comparison, there were 9 weekly deaths from flu in Australia in 2019.

Vaccine effectiveness appears to decrease over time Pouwels et al, [2021], Fowlkes [2021], Puranik et al. [2021]. This is not included in the Doherty model. The result of lower vaccine effectiveness over time would be expected to result in more cases beyond 180 days, the time period which is ignored in the model.

\section{Conclusion}

Fundamental limitations of Doherty Model means that it fails to account for 200,000 or more cases of long covid in children, underestimates death rates by a factor of up to ten, underestimates the severity of the delta variant by a factor of two, fails to make any estimate of potential downside risk, and dramatically overestimates the capabilities of contact tracing in Australia.

\section{Competing interests:}

No competing interests declared.

\section{References}

Doherty Institute. Doherty modelling report revised 10th august 2021, August 2021. URL https://tinyurl.com/ vdvirxx8.

P Huston, VL Edge, and E Bernier. Open science/open data: Reaping the benefits of open data in public health. Canada Communicable Disease Report, 45(11):252, 2019.

Jordana Cepelewicz. The hard lessons of modeling the coronavirus pandemic. Quanta Magazine, pages 1-28, 2021.

Sander Greenland. Interval estimation by simulation as an alternative to and extension of confidence intervals. International Journal of Epidemiology, 33(6):1389-1397, 2004.

Richard F MacLehose and Ghassan B Hamra. Applications of bayesian methods to epidemiologic research. Current Epidemiology Reports, 1(3):103-109, 2014.

Australian Bureau of Statistics. Estimates of aboriginal and torres strait islander australians. abs cat. no. 3238.055.001, 2018. URL https://tinyurl.com/58a2e6dj.

Paul Fine, Ken Eames, and David L Heymann. herd immunity: a rough guide. Clinical infectious diseases, 52(7): 911-916, 2011.

Yiwei Zhang, Karl Johnson, Kristen Hassmiller Lich, Julie Ivy, Pinar Keskinocak, Maria Mayorga, and Julie L Swann. Covid-19 projections for k12 schools in fall 2021: Significant transmission without interventions. medRxiv, 2021.

Nicholas G Davies, Petra Klepac, Yang Liu, Kiesha Prem, Mark Jit, and Rosalind M Eggo. Age-dependent effects in the transmission and control of covid-19 epidemics. Nature medicine, 26(8):1205-1211, 2020.

Vital Surveillances. The epidemiological characteristics of an outbreak of 2019 novel coronavirus diseases (covid-19); china, 2020. China CDC weekly, 2(8):113-122, 2020.

Australian Institute of Health and Welfare. The health and welfare of australia's aboriginal and torres strait islander peoples 2015. cat. no. ihw 147, 2015. URL https://tinyurl.com/nhj8u7rf. 
Alzheimer's Association. Covid-19 associated with long-term cognitive dysfunction, acceleration of alzheimers symptoms I aaic 2021. https://tinyurl. com/tuaax9s7, July 2021. (Accessed on 09/06/2021).

Office for National Statistics. Coronavirus and the social impacts of 'long covid' on peoples lives in great britain: 7 april to 13 june 2021, July 2021. URL https://tinyurl.com/w3zp8k8.

Jacqui Wise. Long covid: One in seven children may still have symptoms 15 weeks after infection, data show, 2021.

Z Hyde, J Parslow, R Quentin Grafton, and T Kompas. What vaccination coverage is required before public health measures can be relaxed in australia?, July 2021. URL https://osf.io/h57dx/.

Stephen Duckett, Danielle Wood, Brendan Coates, Will Mackey, Tom Crowley, and Anika Stobart. Race to 80: our best shot at living with covid. Grattan Institute, 2021.

Koen B Pouwels, Emma Pritchard, Philippa Matthews, Nicole B Stoesser, David W Eyre, Karina-Doris Vihta, Thomas House, Jodie Hay, John Bell, John Newton, et al. Impact of delta on viral burden and vaccine effectiveness against new sars-cov-2 infections in the uk. medRxiv, 2021.

Ashley Fowlkes. Effectiveness of covid-19 vaccines in preventing sars-cov-2 infection among frontline workers before and during b. 1.617. 2 (delta) variant predominance; eight us locations, december 2020-august 2021. MMWR. Morbidity and Mortality Weekly Report, 70, 2021.

Arjun Puranik, Patrick J Lenehan, Eli Silvert, Michiel JM Niesen, Juan Corchado-Garcia, John C OHoro, Abinash Virk, Melanie D Swift, John Halamka, Andrew D Badley, et al. Comparison of two highly-effective mrna vaccines for covid-19 during periods of alpha and delta variant prevalence. medRxiv, 2021. 